\title{
Multiwavelength dissection of a massive heavily dust-obscured galaxy and its blue companion at $z \sim 2$
}

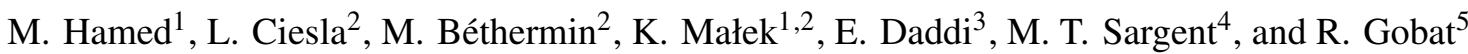 \\ 1 National Centre for Nuclear Research, ul. Pasteura 7, 02-093 Warszawa, Poland \\ e-mail: mahmoud.hamed@ncbj .gov.pl \\ 2 Aix Marseille Univ. CNRS, CNES, LAM, Marseille, France \\ 3 CEA, Irfu, DAp, AIM, Université Paris-Saclay, Université de Paris, CNRS, 91191 Gif-sur-Yvette, France \\ 4 Astronomy Centre, Department of Physics and Astronomy, University of Sussex, Brighton BN1 9QH, UK \\ 5 Instituto de Fisica, Pontificia Universidad Católica de Valparaíso, Casilla 4059, Valparaiso, Chile
}

Received 2 October 2020 / Accepted 13 January 2021

\section{ABSTRACT}

\begin{abstract}
Aims. We study a system of two galaxies, Astarte and Adonis, at $z \sim 2$. At this time, the Universe was undergoing the peak of its star formation activity. Astarte is a dusty star-forming galaxy at the massive end of the main sequence (MS), and Adonis is a less massive companion galaxy that is bright in the ultraviolet and has an optical spectroscopic redshift. We investigate whether this ultramassive galaxy is quenching, and whether it has always been on the MS of star-forming galaxies.

Methods. We used the code CIGALE to model the spectral energy distribution. The code relies on the energetic balance between the ultraviolet and the infrared. We derived some of the key physical properties of Astarte and Adonis, mainly their star formation rates (SFRs), stellar masses, and dust luminosities. We inspected the variation of the physical parameters depending on the assumed dust-attenuation law. We also estimated the molecular gas mass of Astarte from its CO emission, using different $\alpha_{\mathrm{CO}}$ and transition ratios $\left(r_{31}\right)$, and we discuss the implication of the various assumptions on the gas-mass derivation.

Reults. We find that Astarte exhibits a MS-like star formation activity, and Adonis is undergoing a strong starburst phase. The molecular gas mass of Astarte is far lower than the gas fraction of typical star-forming galaxies at $z=2$. This low gas content and high SFR result in a depletion time of $0.22 \pm 0.07 \mathrm{Gyr}$, which is slightly shorter than expected for a MS galaxy at this redshift. The CO luminosity relative to the total infrared luminosity suggests a MS-like activity when we assume a galactic conversion factor and a low transition ratio. The SFR of Astarte is on the same order when different attenuation laws are used, unlike its stellar mass, which increases when shallow attenuation laws are used $\left(\sim 1 \times 10^{11} M_{\odot}\right.$ assuming a Calzetti relation, versus $\sim 4 \times 10^{11} M_{\odot}$ assuming a shallow attenuation law). We discuss these properties and suggest that Astarte might be experiencing a recent decrease in star formation activity and is quenching through the MS following a starburst epoch.
\end{abstract}

Key words. galaxies: evolution - galaxies: high-redshift - galaxies: ISM - infrared: galaxies - submillimeter: galaxies galaxies: starburst

\section{Introduction}

Studying galaxy evolution throughout cosmic time is a key element of modern astrophysics and is crucial for our understanding of the life cycle of the progenitors of passive elliptical galaxies that we observe in the local Universe. Evidence suggests that the star formation rate (SFR) density has peaked around a redshift of $z \approx 2$ (e.g., Hopkins \& Beacom 2006; Madau \& Dickinson 2014; Béthermin et al. 2017; Gruppioni et al. 2020), making this epoch (cosmic noon) particularly interesting. Moreover, at cosmic noon, dusty star-forming galaxies (DSFGs; e.g., Smail et al. 1997; Blain et al. 2002; Weiß et al. 2013a; Casey et al. 2014; Donevski et al. 2020) contributed significantly to the star formation activity of the Universe (e.g., Chapman et al. 2003, 2005). Furthermore, dust-obscured star formation activity plays an important role at higher redshifts (e.g., Takeuchi et al 2005; Murphy et al. 2011; Béthermin et al. 2015; Bourne et al. 2017; Whitaker et al. 2017). It is therefore crucial to study the massive DSFGs at higher redshift.

The plethora of multiwavelength data, especially the infrared (IR) detections from Herschel, played a central role in our understanding of the evolution of DSFGs as a function of redshift. However, there are still controversies regarding how these galaxies build up their stellar masses. These controversies arise from the systematic uncertainties caused by the heavy dust attenuation in this type of object (e.g., Hainline et al. 2011; Michałowski et al. 2012). This is caused by the sensitivity of the stellar mass estimate to the type of star formation history (SFH), the choice of the synthetic stellar population (SSP), and the assumed initial mass function (IMF). The accuracy of the derived stellar masses of DSFGs was also discussed in detail in Casey et al. (2014).

On the other hand, the growing number of Atacama Large Millimeter Array (ALMA) observations in the recent years provides unparalleled help in constraining the evolution of DSFGs. These data allow us to build a comprehensive view of the role of these giant IR-bright sources by tracing their molecular gas and dust content (e.g., Donevski et al. 2020). The wealth of multiwavelength data also contributed to significantly improve the estimation of physical properties that govern such galaxies by modeling their spectral energy distribution (SED, e.g., Burgarella et al. 2005; da Cunha et al. 2008; Noll et al. 2009; Conroy 2013; Ciesla et al. 2014). To build an SED, different aspects of a galaxy must be considered, most importantly, the star formation history (SFH), the change of which strongly affects the derived SFR (e.g., Buat et al. 2014; Ciesla et al. 2017), 
stellar populations of varied ages and metallicities, dust emission with different dust grain sizes and temperatures, nebular and synchrotron emissions, etc. Extinction caused by dust is critically important in any spectrum fitting of a galaxy because it changes the shape of the SED the most by absorbing a significant number of the ultraviolet (UV) photons and thermally reemits them in the IR. This behavior can be modeled by assuming that dust absorbs the shorter wavelength spectrum of galaxies following attenuation laws that are typically described by simple power laws with varying complexities, and it can reproduce the observed extinction in galaxies of different redshifts and types. However, dust attenuation laws are not universal (e.g., Wild et al. 2011; Buat et al. 2018; Małek et al. 2018; Salim \& Narayanan 2020). The need of different attenuation recipes is inevitable in order to reproduce the spectra of galaxies of different masses, IR luminosities, and the redshift. This makes it challenging to interpret some of the physical features, especially when different attenuation laws can reproduce a good SED of a galaxy (Buat et al. 2019).

A non-negligible fraction of galaxies exhibit a nonalignment and sometimes a total disconnection between the dust continuum and the stellar population (Dunlop et al. 2017; Elbaz et al. 2018). This directly challenges SED fitting techniques that rely on the energetic balance between the UV and the IR because the key assumption for these techniques is that any physical property derived from one part of the spectrum should be valid for the entire galaxy. Several approaches have been investigated to test the validity of this strategy. Buat et al. (2019) suggested the decoupling of the stellar continuum from the IR emission by modeling their fluxes in addition to comparing the derived parameters such as the SFRs, dust luminosities, and stellar masses with those derived using full SEDs. Statistical samples of such massive and dusty galaxies (e.g., Dunlop et al. 2017; Elbaz et al. 2018; Buat et al. 2019; Donevski et al. 2020) offer an important insight into the evolution of dust and gas mass through cosmic time. However, the nature of these giants is not fully understood.

The interstellar medium (ISM) is the most important element in understanding the physical processes of star formation itself because it contains the building materials for future stars, most importantly, hydrogen. The hydrogen density was found to be tightly correlated with the SFR, as suggested by Schmidt (1959) and investigated by Kennicutt (1998). This correlation is known as the Schmidt-Kennicutt law, and it takes the gas in its molecular and atomic forms into account, even though molecular gas has the strongest effect. The mass of this gas can be estimated based on the emission of the easily excited CO molecules (e.g., Carilli \& Walter 2013; Weiß et al. 2013b; Decarli et al. 2019; Riechers et al. 2020). Tracing the molecular gas with CO emission relies entirely on already established abundances in galaxies of the local Universe. Large interferometers such as ALMA offer unique opportunities for detecting these emission lines with an unprecedented accuracy. The luminosity of the lines can give an estimate of the molecular hydrogen mass of a galaxy, typically using a conversion factor. On the other hand, conversion factors in high-redshift galaxies are highly debated (see Bolatto et al. 2013 for a comprehensive review).

Nonetheless, an estimate of the molecular gas reservoir of galaxies at the high-mass end of the main sequence (MS) is crucial for characterizing their star formation activity. For instance, Elbaz et al. (2018) showed that some galaxies exhibit a starburst (SB)-like gas depletion timescale although they reside on the MS.

Despite the growing number of detections of these heavily dust-obscured ultramassive objects at high redshift, the progress of SED modeling, and the better comprehension of the highredshift ISM, we still lack a full picture of how these galaxies form and quench. They might always have steadily formed stars throughout the MS, or they might be former SBs transiting to the red sequence through the MS.

To answer these questions, it is essential to understand how the star formation is fueled by the gas in massive objects, and why this activity ceases. Quenching mechanisms are still not fully understood, and they might be caused by active galactic nuclei (AGN) feedback or outflows (e.g., Cattaneo et al. 2009; Dubois et al. 2013; Combes 2017) to environmental effects that can lead to gas-stripping (e.g., Coil et al. 2008; Mendez et al. 2011).

Motivated by these questions, we analyze and interpret the multiwavelength observations of a pair of galaxies at $z \sim 2$ with original COSMOS2015 catalog (Laigle et al. 2016 IDs: 647980 and 648299, hereafter Astarte and Adonis). Astarte is an ultramassive $\left(M_{\star}>10^{11} M_{\odot}\right)$, IR-bright galaxy whose CO emission is serendipitously detected with ALMA. Adonis is a low-mass galaxy bright in near-UV and optical bands.

The structure of this paper is as follows: in Sect. 2 we describe the data of the two galaxies. In Sect. 3.1 we probe the molecular gas of Astarte using its ALMA-detected CO emission line, and in Sect. 3.1.5 we investigate the morphology of this line compared to multiwavelength detections. In Sect. 3.2 we derive the physical properties of the two galaxies using SED fitting. The discussion and conclusion are presented in Sects. 4 and 5, respectively.

Throughout this paper, we adopt the stellar IMF of Chabrier (2003) and $\Lambda$ CDM cosmology parameters (WMAP7, Komatsu et al. 2011), $\mathrm{H}_{0}=70.4 \mathrm{~km} \mathrm{~s}^{-1} \mathrm{Mpc}^{-1}, \Omega_{\mathrm{M}}=0.272$, and $\Omega_{\Lambda}=$ 0.728 .

\section{Observations}

The system of Astarte and Adonis was initially part of a selection of $z \sim 2$ galaxies at the high-mass end $\left(M_{\star}>10^{11} M_{\odot}\right)$ of the MS of star-forming galaxies (e.g., Noeske et al. 2007; Daddi et al. 2010; Rodighiero et al. 2011; Schreiber et al. 2015) detected by Herschel/PACS observations of the COSMOS field (PEP survey, Lutz et al. 2011). In the COSMOS2011 catalog from which the system was selected, the system is not deblended even in the optical and near-IR and appears as a single source. This is probably caused by the fact that this early catalog is mainly built using the $i$ band, in which Astarte is particularly faint. The zCOSMOS survey (Lilly et al. 2009) measured the spectroscopic redshift at the position of the HST/ACS source from zCOSMOS and found $z_{\mathrm{spec}}=2.140$. In the more recent COSMOS catalog (Laigle et al. 2016), both $z$ - and near-IR bands were used to detect and deblend the object. Adonis and Astarte thus have individual flux measurements in the optical bands of Subaru, the near-IR bands of VISTA, and the mid-IR with Spitzer/IRAC. Astarte is detected at 250 and $350 \mu \mathrm{m}$ with Herschel/SPIRE using a $24 \mu \mathrm{m}$ prior (Oliver et al. 2012). The deblending, coupled with the far-IR detection of Astarte, results in a low-mass low-SFR object $\left(\mathrm{SFR}=37 M_{\odot} \mathrm{yr}^{-1}\right.$ with a stellar mass of $9.46 \times 10^{9} M_{\odot}$ ), and a dust-obscured ultramassive object $\left(\mathrm{SFR}=131 M_{\odot} \mathrm{yr}^{-1}\right.$ with a stellar mass of $\left.1.41 \times 10^{11} M_{\odot}\right)$, as initially estimated using LePhare (Arnouts \& Ilbert 2011).

Astarte and Adonis were observed by ALMA as part of a program (2013.1.00914.S, PI: Béthermin) targeting a pilot sample of four massive $z \sim 2$ main-sequence galaxies in band-7 continuum and their $\mathrm{CO}$ emission. The goal was to measure their 


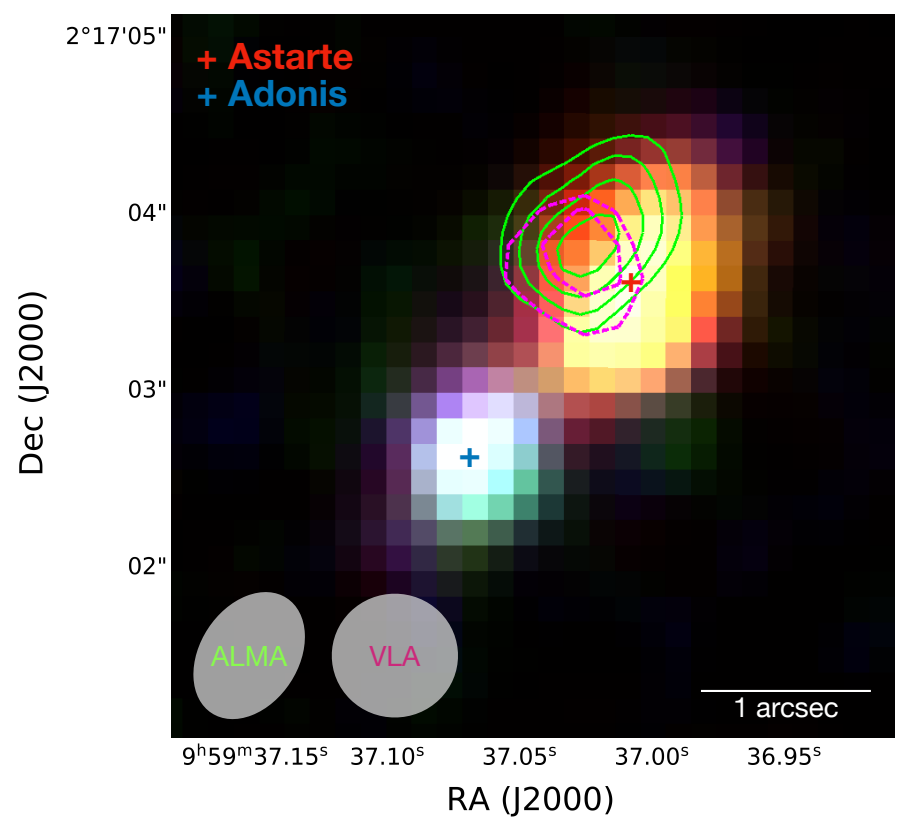

Fig. 1. Integrated flux of ALMA-detected CO emission (green contours) along with the VLA-detected radio continuum at $3 \mathrm{GHz}$ (magenta contours) in the RGB image (VISTA $K s, H$, and $J$ ) of Astarte and Adonis. The beam size of ALMA is $0.78^{\prime \prime} \times 0.50^{\prime \prime}$ (lower left beam). The beam FWHM of VLA is $0.75^{\prime \prime}$. The outermost contour of the CO integrated flux (green) is at $2 \sigma$ significance. The subsequent contours are in steps of $1 \sigma$, and the innermost contour shows $5 \sigma$. The magenta contours show 2 and $3 \sigma$ significance. The blue cross is centered on Adonis, and the red cross is centered on Astarte.

gas and dust content and to compare their short-wavelength morphology with their $\mathrm{CO}$ and continuum morphologies.

\subsection{Near-UV to IR observations}

The near-UV (rest-frame far-UV) detections of our two galaxies are provided by the Canada France Hawaii Telescope (CFHT) in the $u$ band. Visible and near-IR detections (rest-frame near-UV) are obtained via the broad band Suprime-Cam of Subaru in the $B$, $V, r, i^{+}$bands and the mid-IR data (rest-frame near-IR) are from the IRAC camera of Spitzer. The IR-bright Astarte has a MIPS detection at $24 \mu \mathrm{m}$ with a signal-to-noise ratio $(S / N)>20$ and is very bright $(S / N \sim 20)$ in IR detections of Herschel, where the beam size is large. The $100 \mu \mathrm{m}$ observation from PACS did not detect the two galaxies, but provided an upper limit, which is taken into account in the SED fitting because it constrains the far-IR part of the spectrum.

The radio continuum of Astarte was tentatively detected with the Karl G. Jansky Very Large Array (VLA) in the $S$ band at $v=3 \mathrm{GHz}$ (Smolčić et al. 2017). This tentative detection was not included in the initial catalog of Smolčić et al. (2017) because it falls just below their detection threshold of $5 \sigma(S / N=4.3)$. Adonis has not been detected with the VLA at $3 \mathrm{GHz}$. We thus estimated a $3 \sigma$ upper limit from the standard deviation in the cutout image around our two sources. The beam width of the VLA detection is $0.75^{\prime \prime}$, and the continuum is shown in Fig. 1.

The Jin et al. (2018) catalog provides the JCMT fluxes at $850 \mu \mathrm{m}$ for both our galaxies $(2440 \pm 2519 \mu \mathrm{Jy}$ for Astarte and $3910 \pm 2516 \mu \mathrm{Jy}$ for Adonis). We refrain from using these highly deblended fluxes because of the high uncertainties, which are probably caused by the degeneracies in the deblending of this close pair and because most of the flux is unexpectedly attributed
Table 1. Summary of the data of the two sources observed through the different instruments.

\begin{tabular}{lcccc}
\hline \hline & & & Astarte & Adonis \\
\hline COSMOS15 ID & & & 647980 & 648299 \\
\hline Redshift & & & $z_{\text {phot }}=2.153$ & $z_{\text {spec }}=2.140$ \\
\hline Telescope/ & Filter & $\lambda$ & $\begin{array}{c}S_{v} \\
(\mu \mathrm{Jy})\end{array}$ & $\begin{array}{c}S_{v} \\
(\mu \mathrm{Jy})\end{array}$ \\
Instrument & & $(\mu \mathrm{m})$ & $0.104 \pm 0.032$ & $0.492 \pm 0.032$ \\
\hline CFHT/ & $u$ & 0.383 & & \\
MegaCam & & & $0.127 \pm 0.018$ & $0.596 \pm 0.032$ \\
\hline Subaru/ & $B$ & 0.446 & $0.038 \pm 0.049$ \\
Suprime-Cam & $V$ & 0.548 & $0.252 \pm 0.033$ & $0.938 \pm 0.041$ \\
& $r$ & 0.629 & $0.246 \pm 0.029$ & $0.904 \pm 0.073$ \\
& $i^{+}$ & 0.768 & $0.331 \pm 0.035$ & $0.973 \pm 0.042$ \\
& $z^{++}$ & 0.910 & $0.719 \pm 0.062$ & $1.329 \pm 0.063$ \\
\hline VISTA/ & $Y$ & 1.02 & $0.836 \pm 0.155$ & $1.519 \pm 0.162$ \\
VIRCam & $J$ & 1.25 & $2.691 \pm 0.175$ & $2.682 \pm 0.181$ \\
& $H$ & 1.65 & $4.234 \pm 0.241$ & $3.243 \pm 0.254$ \\
& $K s$ & 2.15 & $9.536 \pm 0.351$ & $4.776 \pm 0.362$ \\
\hline Spitzer & IRAC1 & 3.6 & $18.60 \pm 0.07$ & $3.70 \pm 0.10$ \\
& IRAC2 & 4.5 & $25.10 \pm 0.10$ & $2.80 \pm 0.13$ \\
& IRAC3 & 5.8 & $25.10 \pm 2.00$ & $3.60 \pm 2.60$ \\
& IRAC4 & 8.0 & $15.30 \pm 3.30$ & - \\
\hline Spitzer & MIPS1 & 24 & \multicolumn{2}{c}{$351 \pm 17$} \\
\hline Herschel & PACS & 100 & \multicolumn{2}{c}{$<6734$} \\
\hline Herschel & SPIRE & 250 & $17792 \pm 744$ & - \\
& SPIRE & 350 & $16058 \pm 1026$ & - \\
\hline VLA & $S$ & $1.3 \times 10^{5}$ & $9.9 \pm 2.3$ & $<7.3$ \\
\hline & & \multicolumn{3}{c}{} \\
\hline
\end{tabular}

Notes. $S_{v}$ is the flux in $(\mu \mathrm{Jy}) . \lambda$ is the center of the specific filter band.

to the smaller and less IR-bright Adonis. Table 1 presents a summary of the available photometric data from different instruments of the two galaxies.

\subsection{ALMA observation}

Astarte was observed at $2.7 \mathrm{~mm}$ with ALMA (band 3) with a time on source of $45 \mathrm{~min}$ using 32 antennas on September 5, 2015, cycle-2 (P.I. M.Béthermin). We used the Common Astronomy Software Applications package and pipeline (CASA) v5.4 ${ }^{1}$ (McMullin et al. 2007) to flag and reduce the visibility data. The deconvolution was performed with the CLEAN algorithm using natural weighting for an optimal $\mathrm{S} / \mathrm{N}$. The multifrequency synthesis mode of the line-free channels showed an insignificant continuum emission of the spectrum, therefore we did not need to subtract it. In the deconvolution process, the cell size was set to $0.1^{\prime \prime}$. The achieved synthesized beam size is $0.78^{\prime \prime} \times 0.56^{\prime \prime}$, the velocity resolution of the cube is $21.36 \mathrm{~km} \mathrm{~s}^{-1}$, and the rms is $0.47 \mathrm{mJy}_{\text {beam }}^{-1} \mathrm{~km} \mathrm{~s}^{-1}$ per channel.

\section{Results}

\subsection{Probing the molecular gas of Astarte}

In the data cube we find only one significant line and no significant continuum source in the field of view. The line extraction procedure along with the derivation of the luminosity and the gas mass are described in the following subsections.

\subsubsection{Line extraction}

The ALMA-detected emission line of Astarte corresponds to the $\mathrm{CO}(3-2)$, with a peak at an observed frequency of

\footnotetext{
1 https://casa.nrao.edu/
} 


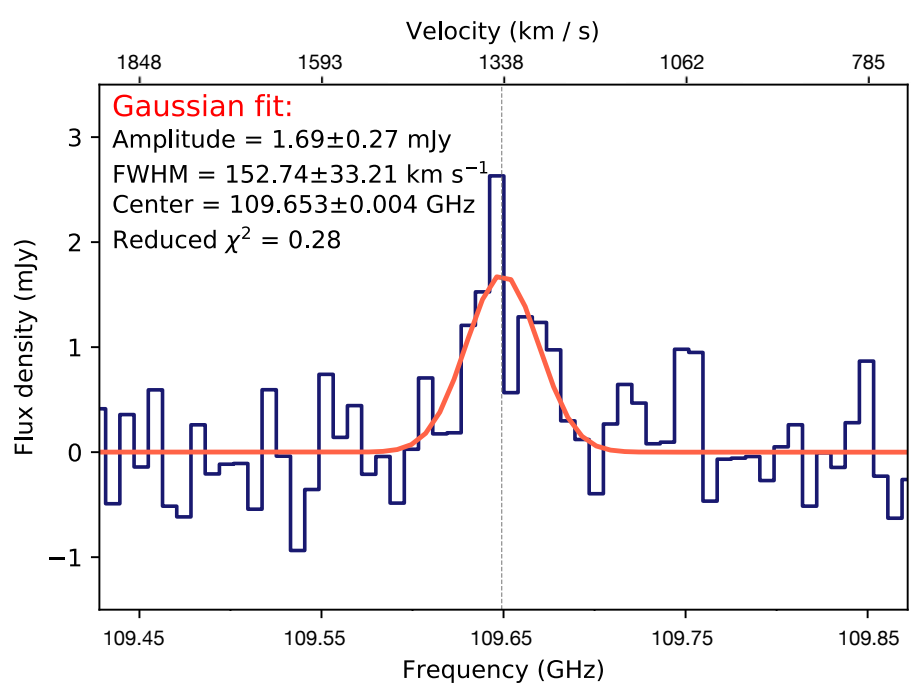

Fig. 2. Spectral profile of Astarte with the redshifted $\mathrm{CO}(3 \rightarrow 2)$ line (dashed vertical gray line) and the Gaussian fit (red) with its properties.

$v_{\text {obs }}=109.65 \mathrm{GHz}$, implying $z_{\mathrm{CO}(3-2)}=2.154$, which agrees with the photometric redshift $z_{\text {phot,Astarte }}=2.153_{-0.058}^{+0.051}$ Laigle et al. (2016). This confirms Astarte as the origin of the detected$\mathrm{CO}$ emission. We do not detect Astarte in the continuum and measured a $3 \sigma$ upper limit from the map of $0.117 \mathrm{mJy}$. The expected flux densities from the SED modeling discussed in Sect. 3.2.6 are $0.007 \mathrm{mJy}$ and $0.049 \mathrm{mJy}$ for Adonis and Astarte, respectively. It is thus not surprising that none of our two sources are detected. The flux uncertainty was determined by deriving the standard deviation in the source-free pixels in the map that was not corrected for the primary beam because it has similar noise levels in the emission-free pixels (the noise in the central region is $\sim 2 \%$ higher than in the outermost region of the map). The achieved $\mathrm{S} / \mathrm{N}$ is 5.2 for the brightest channel of the $\mathrm{CO}(3-2)$ of Astarte. The emission line was extracted by fitting a Gaussian to the profile. The goodness of the Gaussian fit was verified with a $\chi^{2}$ test. Its properties are summarized in Fig. 2 along with the redshifted $\mathrm{CO}(3-2)$ line. The full width at half maximum (FWHM) of the Gaussian is found to be $152.74 \pm 33.21 \mathrm{~km} \mathrm{~s}^{-1}$.

The spectroscopic redshift of the system at the position of HST detection found by by the zCOSMOS survey Lilly et al (2009) is $z_{\mathrm{spec}}=2.140$. This $z_{\mathrm{spec}}$ corresponds to that of Adonis because only this UV-bright galaxy is detected with HST/ACS. When the redshift difference of Astarte and Adonis is taken into account, the corresponding radial velocity difference $\Delta V$ is $1335 \mathrm{~km} \mathrm{~s}^{-1}$. This velocity difference is greater than what is found in interacting pairs of galaxies, which is typically $\Delta V<$ $350 \mathrm{~km} \mathrm{~s}^{-1}$ (Lambas et al. 2003; Alonso et al. 2004). Outflows and absorption in the UV lines could account for few hundred $\mathrm{km} \mathrm{s}^{-1}$ (Cassata et al. 2020), or a division of the Hubble flow and peculiar motions, which could account for a significant velocity contribution if it is along the line of sight. Therefore this does not rule out a possible interaction between Astarte and Adonis.

\subsubsection{Line-integrated flux and luminosity}

The intensity is calculated by integrating over the Gaussian fit of the line, which is then converted into the apparent line luminosity $\left(L^{\prime}\right)$ using the expression from Solomon et al. (1997), which expresses $L^{\prime}$ with the integrated source brightness temperature
Table 2. Summary of the $\mathrm{CO}(3-2)$ emission line properties of Astarte.

\begin{tabular}{cccc}
\hline \hline $\begin{array}{c}\text { Peak flux } \\
\text { density (mJy) }\end{array}$ & $\begin{array}{c}I_{\mathrm{CO}(3-2)}^{\mathrm{spec}}\left(\mathrm{Jy} \mathrm{km} \mathrm{s}^{-1}\right) \\
I_{\mathrm{CO}(3-2)}^{\mathrm{mom}}\end{array}$ & $\begin{array}{c}L_{\mathrm{CO}(3-2)}^{\prime} \\
\left(10^{9} \mathrm{~K} \mathrm{~km} \mathrm{~s}^{-1} \mathrm{pc}^{2}\right)\end{array}$ \\
\hline $1.690 \pm 0.277$ & $0.251 \pm 0.062$ & $0.328 \pm 0.047$ & $8.508 \pm 1.219$ \\
\hline
\end{tabular}

Notes. $I_{\mathrm{CO}(3-2)}^{\mathrm{spec}}$ is achieved by integrating over the Gaussian of the emission line. $I_{\mathrm{CO}(3-2)}^{\mathrm{mom}}$ is the intensity derived from the moment-zero map.

in units of $\mathrm{K} \mathrm{km} \mathrm{s}^{-1} \mathrm{pc}^{2}$,

$$
L_{\text {line }}^{\prime}=3.25 \times 10^{7} \times I \times \frac{D_{L}^{2}}{(1+z)^{3} v_{\text {obs }}^{2}},
$$

where $D_{\mathrm{L}}$ is the luminosity distance in Mpc, $I$ is the intensity in $\mathrm{Jy} \mathrm{km} / \mathrm{s}$, and $v_{\mathrm{obs}}$ is the observed frequency in GHz. As a consistency test, we also estimated the integrated flux of the line using the moment-zero map, which was obtained by summing the channels in which the emission line is detected. The line flux is measured in the moment-zero map using a 2D Gaussian fit of the source. As shown in Béthermin et al. (2020), there is no significant difference between this method and a fit in the uv plane for faint compact sources observed by ALMA. The resulting flux densities of the two methods are presented in Table 2. There is $1.2 \sigma$ significant difference between the intensities derived by each methods. The spectrum is extracted at a single point assuming a point source, while the $2 \mathrm{D}$ fit can recover the flux from an extended source. This small difference in flux suggests that our source was marginally resolved. Hereafter, we use the flux from the moment-zero map, which takes this into account. However, we cannot formally exclude another faint and diffuse component at larger scale considering the depth of our data. Figure 1 shows the flux-integrated moment-zero map of Astarte represented by confidence levels contours. The size of the $\mathrm{CO}$ disk is $\sim 74 \mathrm{kpc}$.

\subsubsection{Deriving the molecular gas mass}

To derive the total mass of the molecular gas in a galaxy, we assumed that the $\mathrm{H}_{2}$ mass is proportional to the $\mathrm{CO}(1-0)$ line luminosity, which is the commonly used tracer of the cold starforming molecular clouds because only a small excitation potential is required. The $\mathrm{H}_{2}$ mass can be derived using a conversion factor $\alpha_{\mathrm{CO}}$ (e.g., Downes \& Solomon 2003; Greve et al. 2005; Tacconi et al. 2006; Carilli \& Walter 2013; Bothwell et al. 2013a),

$M_{\mathrm{H}_{2}}=\alpha_{\mathrm{CO}} L_{\mathrm{CO}(1-0),}^{\prime}$

where $M_{\mathrm{H}_{2}}$ is the mass of the molecular hydrogen in $M_{\odot}, \alpha_{\mathrm{CO}}$ is the conversion factor, and $L_{\mathrm{CO}(1-0)}^{\prime}$ is the line luminosity in $\mathrm{K} \mathrm{km} \mathrm{s}^{-1} \mathrm{pc}^{2}$. The practice of deriving the $\mathrm{H}_{2}$ mass with this method is very common, especially for galaxies at high redshifts, for which information and spatial resolution is often limited. Our $\mathrm{CO}(3-2)$ line luminosity has to be converted into $\mathrm{CO}(1-0)$ luminosity using a luminosity line ratio $r_{31}=L_{\mathrm{CO}(3-2)}^{\prime} / L_{\mathrm{CO}(1-0)}^{\prime}$. We used $r_{31}=0.42 \pm 0.07$, which is the average ratio found for $z=1.5$ SFGs by Daddi et al. (2015). This resulted in

$L_{\mathrm{CO}(1-0)}^{\prime}=(2.03 \pm 0.59) \times 10^{10} \mathrm{~K} \mathrm{~km} \mathrm{~s}^{-1} \mathrm{pc}^{2}$.

To convert this luminosity into hydrogen mass, we used two conversion factors: $\alpha_{\mathrm{CO}}=0.8$, and a galactic conversion factor of $\alpha_{\mathrm{CO}}=4.36$. The first recovered the molecular gas mass in SBs 

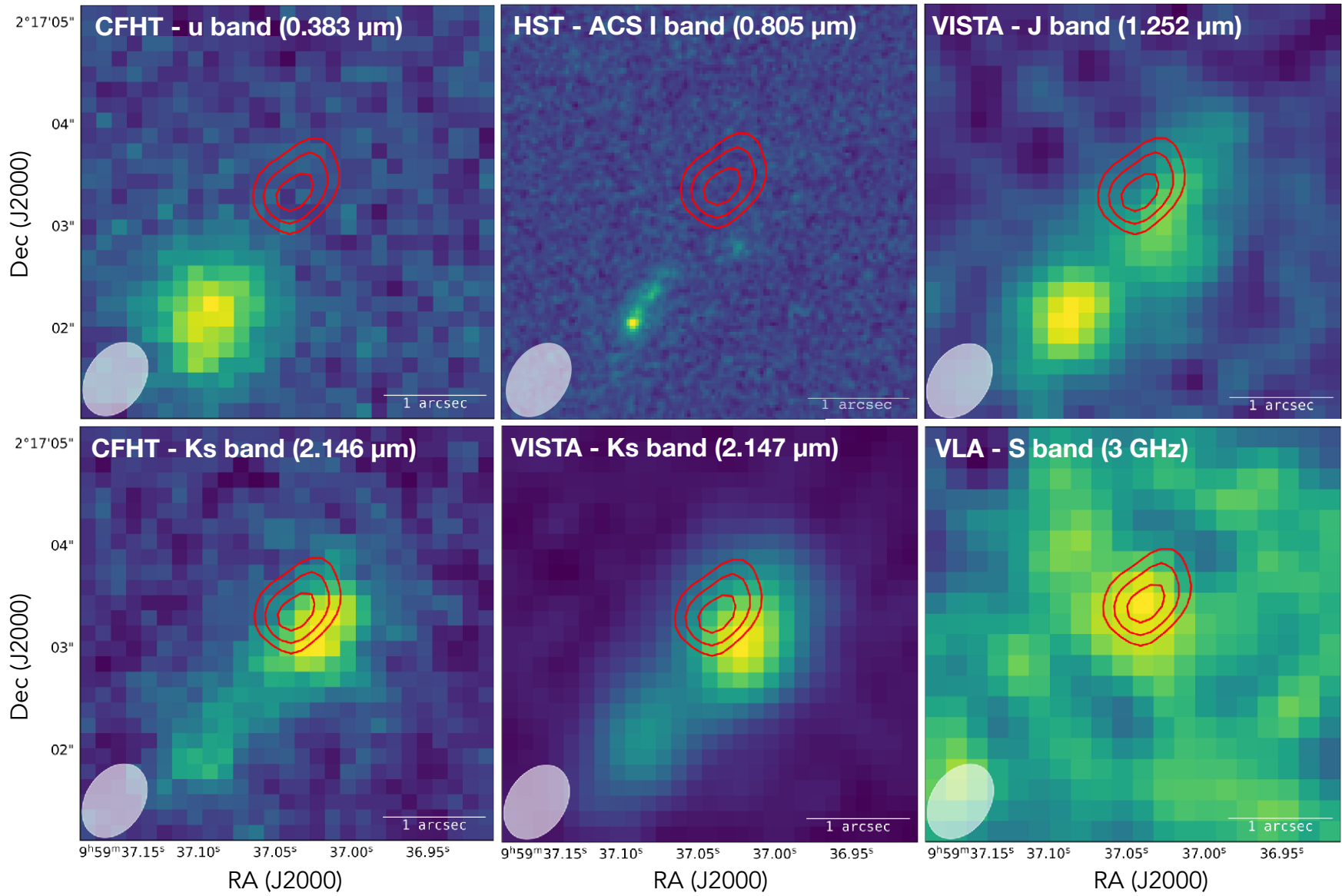

Fig. 3. ALMA-detected $\mathrm{CO}(3-2)$ emission line contour map (red contours) of Astarte overlaid on detections from different telescopes and instruments at different bands as specified in every panel. From upper left to lower right: CFHT $U$ band at $0.383 \mu \mathrm{m}$. HST I band at $0.805 \mu \mathrm{m}$. VISTA $J$ band at $1.252 \mu \mathrm{m}$. CFHT $K s$ band at $2.146 \mu \mathrm{m}$. VISTA $K s$ band at $2.147 \mu \mathrm{m}$ and for the VLA detection at $3 \mathrm{GHz}$. The outermost contour is $3 \sigma$, and the subsequent contours are in steps of $1 \sigma$ with red innermost contour showing $5 \sigma$. The beam size is $0.78^{\prime \prime} \times 0.50^{\prime \prime}$. The white bar shows the 1 arcsec scale.

and submillimeter galaxies, where the gas is efficiently heated by dust. The galactic conversion factor is suitable for normal MS galaxies (Downes \& Solomon 1998; Bolatto et al. 2013; Carilli $\&$ Walter 2013). For $\alpha_{\mathrm{CO}}=0.8$, the mass of the molecular hydrogen is $M_{\mathrm{H}_{2}(\alpha=0.8)}=(1.62 \pm 0.47) \times 10^{10} M_{\odot} . \alpha_{\mathrm{CO}}=4.36$ results in $M_{\mathrm{H}_{2}}=(8.85 \pm 2.57) \times 10^{10} M_{\odot}$, which is a five times larger gas reservoir than we derived with $\alpha_{\mathrm{CO}}=0.8$.

\subsubsection{Dynamical mass}

With the velocity FWHM of the $\mathrm{CO}(3-2)$ line, we used the method described in Bothwell et al. (2013b) to estimate the dynamical mass of Astarte. Assuming that a rotating disk is the origin of the detected line, the dynamical mass can be written as in Neri et al. (2003),

$M_{\text {dyn }}\left(M_{\odot}\right)=4 \times 10^{4} \Delta V^{2} R / \sin ^{2}(i)$,

where $\Delta V$ is the FWHM of the line velocity, $i$ is the inclination angle of the disk, and $\mathrm{R}$ is the radius of the disk in kiloparsec. For a random inclination of $\langle i\rangle=57.3^{\circ}$ (Law et al. 2009), the dynamical mass is found to be $(1.11 \pm 0.23) \times 10^{11} M_{\odot}$. The ratio of gas mass to dynamical mass for a galactic conversion factor is therefore $M_{\mathrm{H}_{2}} / M_{\text {dyn }}=0.76 \pm 0.33$. For $\alpha_{\mathrm{CO}}=0.8$, $M_{\mathrm{H}_{2}} / M_{\mathrm{dyn}}=0.15 \pm 0.07$. The hydrogen mass derived assuming a galactic conversion factor is able to trace the dynamical mass of Astarte, despite the relatively low FWHM of the CO line, as it is for similar FWHM values in Bothwell et al. (2013a).

\subsubsection{CO emission morphology}

We investigated the morphology of the $\mathrm{CO}(3-2)$ emission line of Astarte in relation to other wavelength detections of the system to closely study the association of the $\mathrm{CO}$ component with the UV, optical, and IR components, as shown in Fig. 3. HST's observation in the $I$ band (rest-frame mid-UV) do not show Astarte because it is heavily obscured by dust. However, the young stellar population of the less-dusty Adonis is visible in the HST $I$ band and is bright in the $u$-band detection of CFHT (restframe far-UV) and in the $J$ band of VISTA (rest-frame near-UV). In the $K s$ bands of CFHT and VISTA, which correspond to restframe visible light, Adonis becomes less bright and is very faint at higher wavelength observations of ALMA and VLA.

The dusty Astarte is not visible in the $u$ band of CFHT (restframe far-UV). It is detected in the $K s$ bands of VISTA and CFHT, however, showing a bright stellar population in the visible rest-frame wavelengths. A spatial offset (of $\sim 0.39^{\prime \prime}$ ) is visible between the ALMA-detected CO emission and the emission of the stellar population (observed in the $K s$ bands) of Astarte. Faisst et al. (2020) found an average offset between the COSMOS2015 catalog and the Gaia reference frame of $\Delta(\mathrm{RA})=$ $-63.9_{-60.2}^{+70.7}$ milliarcsec and $\Delta(\mathrm{Dec})=-1.4_{-67.3}^{+80.4}$ milliarcsec. This 
systematic offset cannot explain the visible offset between the $\mathrm{CO}$ emission and the rest-frame optical counterparts of Astarte. Moreover, we show that the continuum detected by the VLA at $3 \mathrm{GHz}$ of Astarte and its $\mathrm{CO}$ emission detected by ALMA are aligned, eliminating the possibility of a systematic error due to the synthesized beam size of ALMA.

Although the original spectroscopic redshift of 2.140 (for both sources) found by zCOSMOS (Lilly et al. 2009) was derived from the visible range of the HST observation, in which Astarte is not observed, ALMA offers a spectroscopic redshift for the latter $\left(z_{\mathrm{ALMA}}=2.154\right)$. This shows the importance of long-wavelength detections especially for dust-obscured galaxies where the UV to near-IR emission is heavily attenuated (Schreiber et al. 2018a; Wang et al. 2019).

\subsection{SFRs, stellar masses, and dust luminosities}

\subsubsection{SED modeling}

We used the SED modeling code CIGALE$^{2}$ (Boquien et al. 2019) to derive the physical properties of our sources. The code allows modeling galaxy SED from the UV to the radio wavelengths, taking the energetic balance between the emission absorbed by dust in the UV-visible range and the IR emission into account. CIGALE offers a variety of modules for each physical process a galaxy may undergo. The modules that we used in our SED fitting procedures are described below.

\subsubsection{Stellar component}

To model the stellar component of Astarte and Adonis, we used the SSP of Bruzual \& Charlot (2003). This stellar library computes the direct stellar contribution to the spectrum (UV to nearIR range) by populating the galaxy with young and old stars of different masses, as well as the required gas mass that will produce such population. This model was developed based on observations of nearby stellar populations, and it describes the various stellar emissions that are expected in encounters within any galaxy. These models depend on the metallicity and the separation age ${ }^{3}$. We used a solar-like metallicity and took nebular emission into account because they contribute to the total SED model from the UV to near-IR.

Different stellar demographics must be modeled with an appropriate SFH in any SED modeling because it is critical to estimate the contribution of the young and old stars to the total flux. An appropriate SFH is key to deriving the SFR of a galaxy because it strongly depends on the assumptions made (Ciesla et al. 2017). CIGALE offers different SFH scenarios that vary from the simple delayed SFH to more complex scenarios containing episodes of bursts or sudden drops in SFRs. We used the SFH proposed by Ciesla et al. (2017), which is a combination of a smooth delayed buildup of the stellar population to model the long-term SFH of a galaxy, and a recent flexibility in the past few hundred million years to allow for recent and drastic SFR variations (burst or quench). This SFH model has been proven to limit biases by decoupling the estimations of the stellar mass, which are mainly constrained by rest frame near-IR data, from the SFR, which is constrained by UV and IR data (e.g., Ciesla et al. 2016, 2018; Schreiber et al. 2018b,a). This type of SFH was used in the study of high-redshift $(z<3)$ passive galaxies to model their SED (e.g., Schreiber et al. 2018b,a; Merlin et al.

\footnotetext{
http://cigale.lam.fr

Age of the separation between the young and old stellar population.
}

2018). We limited the recent burst or quench episodes to the last $100 \mathrm{Myr}$ of the life of our sources. The recent burst is motivated by the ALMA detection, but it is important to note that this burst makes it difficult to constrain the past SFH. The burst part of the SFH is usually responsible for fitting the UV data, whereas the previous SFH (delayed) is driven by the older stellar population, manifested in the visible part of the SED.

\subsubsection{Attenuation laws}

Two prominent attenuation laws are those of Calzetti et al. (2000, hereafter C00) and Charlot \& Fall (2000, hereafter CF00). They are widely used in the literature, and along with their alternations, they can describe the behavior of the extinction in the UV to near-IR caused by dust.

$\mathrm{COO}$ and their recipes are at their core equivalent to reducing the short-wavelength flux from a stellar population by an opaque screen, with the opacity being dependent on the total extinction of the stellar emission at the $B$ and $V$ bands.

Another approach is the CF00 power law, which is fundamentally different from that of $\mathrm{C} 00$ : it attributes a different attenuation to the ISM and to the birth clouds (BC). This makes the dust more effective at absorbing the UV light because the young stellar emission has to pass through the dust in the BC and the ISM. Stars that are older are only attenuated by the ISM dust. The CF00 approach is slightly more complex and physical than that of $\mathrm{COO}$ for high-redshift ultradusty galaxies, embodying different dust distributions and densities throughout a galaxy.

C00 and CF00 relied for their efficiency of attenuating the stellar population on power laws for their slopes. The power-law slopes for BCs and ISM in CF00 were originally fixed at -0.7 each. The recipe of Lo Faro et al. (2017, hereafter LF17) of CF00 was tuned by assuming a power law for the slope of the attenuation in the ISM equal to -0.48 . This recipe provides a steeper attenuation curve at shorter wavelengths. We used these three attenuation laws and compared their best fits and their effects on deriving the physical properties of our sources.

To assess which attenuation laws to use when different modules can produce good and comparable fits, we employed the Bayesian information criterion (BIC), defined as the $\chi^{2}+k \ln n$, where $\chi^{2}$ is the unreduced goodness of the fit, $k$ is the degree of freedom of the model, and $n$ is the total number of photometric fluxes used in the fit of the galaxy. We then evaluated the preference of a model over another by calculating the difference between their BICs: $\triangle \mathrm{BIC}>2$ translates into a notable difference between the two laws, and the fit with the lowest $\chi^{2}$ is preferred. This method was used by Ciesla et al. (2018) to choose successful scenarios of SFHs of quenching galaxies, and by Buat et al. (2019) to assess SEDs of $z \sim 2$ ALMA-detected galaxies.

\subsubsection{Dust emission}

To model the dust emission, we used the Draine et al. (2014) IR emission models, which were calibrated using high-resolution observation of the Andromeda galaxy. Draine et al. (2014) considered a variety of dust grains heated by different intensities coming from the stars and the photodissociation regions, and this is an improved version of the previous Draine \& $\mathrm{Li}$ (2007) model by varying the dust opacity across the radius of a galaxy. This IR model was successful in reproducing dust emissions of millions of Herschel-detected galaxies as a part of the HELP project (Małek et al. 2018). 
Table 3. Input parameters used to fit the SEDs of Astarte and Adonis with CIGALE.

\begin{tabular}{|c|c|c|}
\hline Parameter & & Values \\
\hline \multicolumn{3}{|c|}{ Star formation history Ciesla et al. (2017) } \\
\hline Stellar age ${ }^{(i)}$ & age $_{\text {main }}$ & $0.8-3.2 \mathrm{Gyr}$ by a bin of $0.2 \mathrm{Gyr}$ \\
\hline e-folding time ${ }^{(i i)}$ & $\tau_{\text {main }}$ & $0.8,1,3,5,8 \mathrm{Gyr}$ \\
\hline Age of burst/quench episode & $t_{\text {flex }}$ & $5,10,50,100 \mathrm{Myr}$ \\
\hline SFR ratio after/before & $r_{\mathrm{SFR}}$ & $10^{-2}, 10^{-1}, 0,10^{1}, 10^{2}, 10^{3}$ \\
\hline \multicolumn{3}{|c|}{ Stellar synthesis population (Bruzual \& Charlot 2003) } \\
\hline Initial mass function & IMF & (Chabrier 2003) \\
\hline Metallicity & $Z$ & 0.02 \\
\hline Separation age & & $10 \mathrm{Myr}$ \\
\hline \multicolumn{3}{|c|}{ Dust attenuation laws } \\
\hline \multicolumn{3}{|c|}{ (Calzetti et al. 2000) } \\
\hline Colour excess of young stars & $E(B-V)$ & $0.1-1$ by a bin of 0.1 \\
\hline Reduction factor $^{(i i i)}$ & $f_{\text {att }}$ & $0.3,0.5,0.8,1.0$ \\
\hline \multicolumn{3}{|c|}{ (Charlot \& Fall 2000), (Lo Faro et al. 2017) } \\
\hline$V$-band attenuation in the ISM & $A_{V}^{\mathrm{ISM}}$ & $0.3-3$ by a bin of 0.1 \\
\hline$A v_{V}^{\mathrm{ISM}} /\left(A_{V}^{B C}+A_{V}^{\mathrm{ISM}}\right)$ & $\mu$ & $0.3,0.5,0.8,1$ \\
\hline Power law slope of the ISM & & $-0.7,-\mathbf{0 . 4 8}$ \\
\hline Power law slope of the BC & & -0.7 \\
\hline \multicolumn{3}{|c|}{ Dust emission model (Draine et al. 2014) } \\
\hline Mass fraction of PAH & $q_{\mathrm{PAH}}$ & $1.77,2.50,3.19$ \\
\hline Minimum radiation field & $U_{\min }$ & $10,25,30,40$ \\
\hline Power law slope & $\alpha$ & 2 \\
\hline \multicolumn{3}{|c|}{ Synchrotron emission } \\
\hline Far-IR/radio correlation coefficient & & $2.3-2.9$ by a bin of 0.1 \\
\hline Power law slope slope & $\alpha_{\text {synchrotron }}$ & $0.4-0.9$ by a bin of 0.1 \\
\hline
\end{tabular}

Notes. ${ }^{(i)}$ The stellar age is the age of the main stellar population. ${ }^{(i)}$ The e-folding time is the time required for the assembly of the majority of the stellar population. ${ }^{\text {(iii) }}$ The reduction factor $f_{\text {att }}$ is the color excess in old relative to young stars.

\subsubsection{Synchrotron emission}

The VLA detection at $3 \mathrm{GHz}$ of Astarte and Adonis allowed us to model the synchrotron emission of our objects, taking a nonthermal power law of the synchrotron spectrum and the ratio of the far-IR/radio correlation into account. The different parameters we used to build our SEDs are shown in Table 3.

\subsubsection{SED fitting results}

In the case of Astarte, the $\mathrm{CF} 00$ and LF17 attenuation laws result in best SED fits over C00. The BIC of every model was calculated and is shown in Table 4 along with the other quality of fit assessments. $\Delta \mathrm{BIC}_{(\mathrm{C} 00, \mathrm{CF} 00)}=21.88$ and $\Delta \mathrm{BIC}_{(\mathrm{CF} 00, \mathrm{LF} 17)}=2.29$, this privileged the best fit produced with the LF17 attenuation law and therefore was taken into account in deriving the physical properties. Despite the uncertainties on any assumed SFH model, the adopted SFH here fit the short wavelength data best.

For the less-massive Adonis, CF00 gave overall better fits than $\mathrm{C} 00$ and $\mathrm{LF} 17$, with $\triangle \mathrm{BIC}_{(\mathrm{LF17}, \mathrm{CF} 00)}=3.48$ and $\triangle \mathrm{BIC}_{(\mathrm{C} 00, \mathrm{CF} 00)}=5.77$. The best SEDs of Astarte and Adonis are shown in Fig. 4. The signature of dust attenuation is clear in the two SEDs, where the heavily dust-obscured Astarte has more attenuation of its overall stellar mass than is the case with Adonis. The derived properties of both galaxies are shown in Table 5 . The $L_{\mathrm{IR}}$ of Astarte of about $10^{12}$ qualifies it to be an ultraluminous IR galaxy (ULIRG), while the low dust content of Adonis is manifested in the weaker IR luminosity and lower dust mass.

To closely inspect the visible dissociation of the gas and the stellar population in Astarte, we followed the method used
Table 4. Comparison between the quality of fits of Astarte and Adonis produced with CIGALE with the three attenuation laws.

\begin{tabular}{ccccc}
\hline \hline & Attenuation law & $\chi^{2}$ & Reduced $\chi^{2}$ & BIC \\
\hline \multirow{4}{*}{ Astarte } & C00 & 43.22 & 2.12 & 73.18 \\
& CF00 & 18.34 & 0.97 & 51.30 \\
& LF17 & 16.06 & 0.84 & 49.01 \\
\hline \multirow{3}{*}{ Adonis } & C00 & 16.28 & 1.10 & 46.78 \\
& CF00 & 10.51 & 0.70 & 41.01 \\
& LF17 & 13.99 & 0.93 & 44.49 \\
\hline
\end{tabular}

in Buat et al. (2019) by dissecting the stellar continuum and the IR emission and comparing their derived properties with those obtained using full SEDs. Taking the UV to near-IR data $(0.3-8 \mu \mathrm{m})$ into account, the best fit for the stellar continuum was obtained with the $\mathrm{C} 00$ law, with $\Delta \mathrm{BIC}_{(C F 00, C 00)}=8.3$ and $\triangle \mathrm{BIC}_{(L F 17, C 00)}=2.7$. The better quality fit of the stellar continuum produced using $\mathrm{C} 00$ is expected because this power law effectively attenuates the young stellar population, while the other two laws can be equally efficient in attenuating the older stars, a behavior that translates into a rise in the nearIR absorbed light and therefore a rise in the total IR emission. The IR luminosity derived from the stellar continuum gives $L_{\text {dust }}=(2.43 \pm 1.01) \times 10^{12} L_{\odot}$, relatively close to $L_{\text {dust }}$ derived from the full SED. The stellar mass derived from the stellar emission gives $(1.3 \pm 0.2) \times 10^{11} M_{\odot}$ and the $\mathrm{SFR}_{(\mathrm{UV}-\text { near-IR })}=$ 

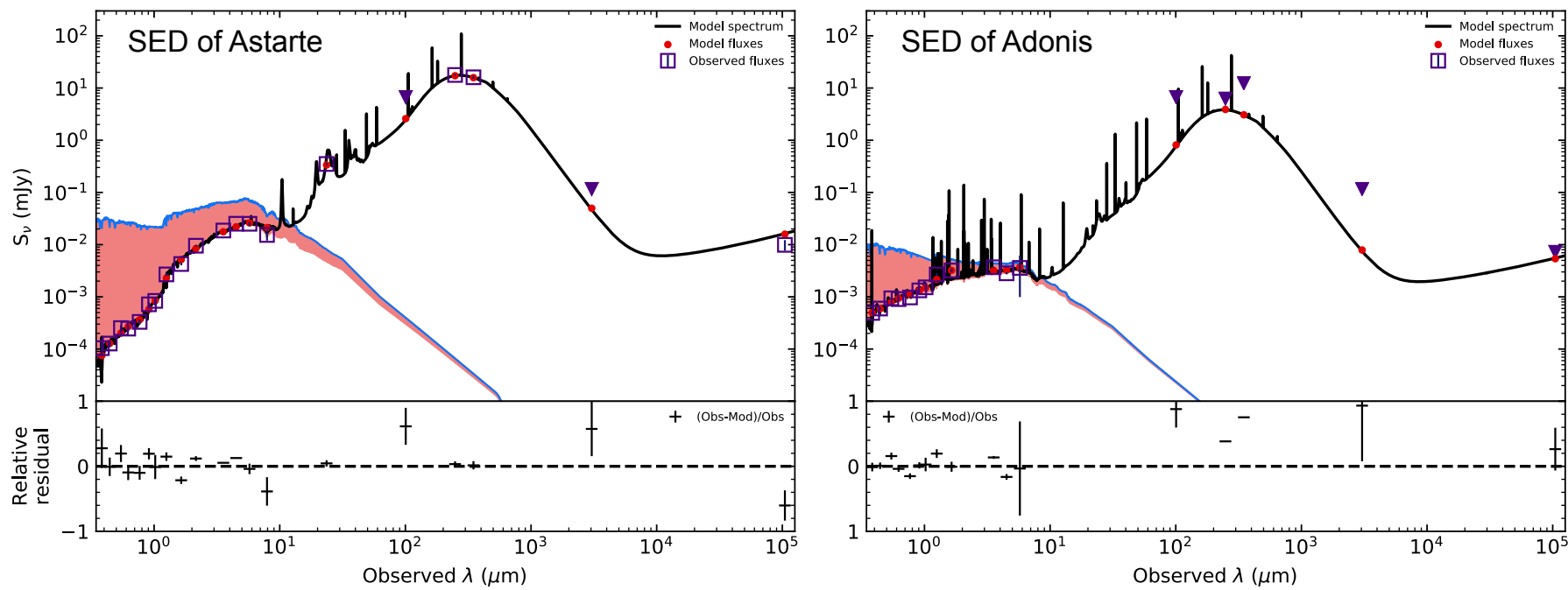

Fig. 4. Best fits of the constructed SEDs of Astarte and Adonis along with their relative residuals. The SED of Astarte (left) is produced using the LF17 attenuation law. The SED of Adonis (right) is produced using the CF00 attenuation law. The best fit is shown in black. The unattenuated stellar emission is shown with the blue line. The filled region shows the difference between the unattenuated and the attenuated stellar emission, absorbed by dust. Red dots are the best-fit values of the observations, which are shown with the purple boxes. Upper limits are shown as purple triangles.

Table 5. Summary of the physical properties obtained for Astarte and Adonis obtained with CIGALE.

\begin{tabular}{lcc}
\hline \hline Physical property & Astarte & Adonis \\
\hline Redshift & $z_{\mathrm{CO}}=2.154$ & $z_{\text {spec }}=2.140$ \\
$L_{I R}\left(10^{12} L_{\odot}\right)$ & $3.16 \pm 0.06$ & $0.62 \pm 0.04$ \\
$\mathrm{SFR}\left(M_{\odot} \mathrm{yr}^{-1}\right)$ & $395 \pm 20$ & $129 \pm 59$ \\
$M_{\star}\left(M_{\odot}\right)$ & $(3.74 \pm 0.19) \times 10^{11}$ & $(9.37 \pm 1.76) \times 10^{9}$ \\
$M_{\text {dust }}\left(10^{9} M_{\odot}\right)$ & $1.01 \pm 0.11$ & $0.86 \pm 0.13$ \\
\hline
\end{tabular}

$430 M_{\odot} \mathrm{yr}^{-1}$. From the IR data (MIPS - ALMA continua), we obtain $L_{\text {dust }}=(3.25 \pm 0.08) \times 10^{12} L_{\odot}$, consistent with the luminostiy derived with the full SED. This result agrees with the results of Buat et al. (2019), who they found consistent dust luminosities derived from both the full SED and the IR data, while $L_{\text {dust }}$ deduced from the stellar continuum was underestimated.

\section{Discussion}

Figure 5 shows the relative position of our galaxies to the MS of Schreiber et al. (2015). Adonis lies on $10 \times \mathrm{MS}$, qualifying it to be a strong SB, despite its relatively low SFR. While being a SB, this type of source cannot be detected even by the deepest $3 \mathrm{~mm}$ ALMA survey (expected flux of $7 \mu \mathrm{Jy}$ ), which have a $1 \sigma$ noise of $9.7 \mu$ Jy González-López et al. (2020).

Astarte is a MS galaxy with all the different attenuation recipes used. However, there is a clear difference concerning the position of Astarte relative to the MS as a result of the three attenuation laws. This is attributed to the significant difference in the derived stellar masses, with CF00 and LF17 attenuation laws resulting in a higher stellar mass than $\mathrm{C} 00$ as a result of the highest attenuation in near-IR. This contributes to a lower specific SFR $\left(\mathrm{sSFR}=\mathrm{SFR} / M_{\star}\right.$ ) because SFRs do not differ significantly with the three laws.

The host halo masses of $z \sim 2$ Herschel-detected massive MS galaxies were investigated in Béthermin et al. (2014) using

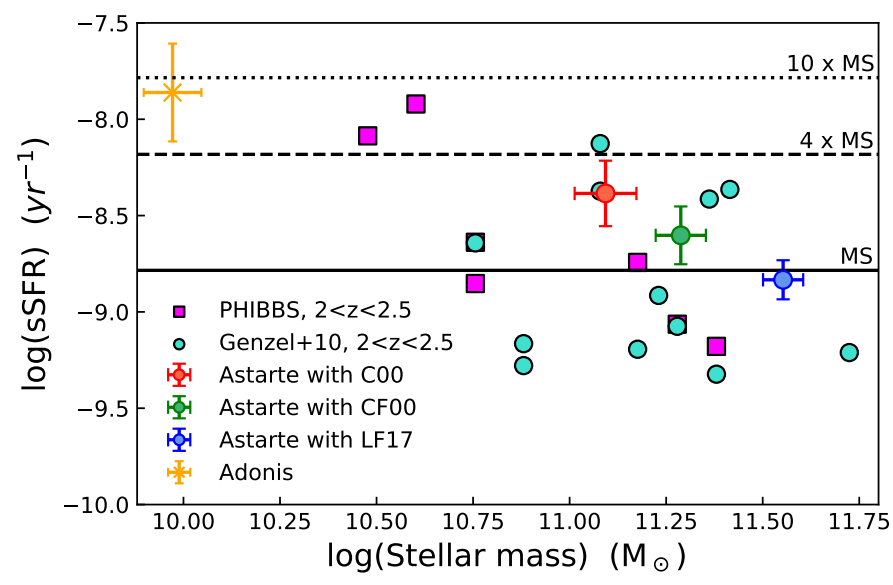

Fig. 5. Relative position of the $\operatorname{sSFR}\left(\mathrm{SFR} / M_{\star}\right)$ and stellar mass of Astarte using the three attenuation laws to the main sequence of Schreiber et al. (2015) at $z=2$. The yellow star shows the relative position of Adonis to the MS. Magenta squares denote PHIBSS COdetected SFGs at $z \approx 2$ (Tacconi et al. 2013). Turquoise circles are ULIRGs at $2<z<2.5$ from Genzel et al. (2010). The solid line shows the MS of Schreiber et al. (2015). The dashed and dotted lines are MS $\times 4$ and $\mathrm{MS} \times 10$, respectively.

clustering and X-ray stacking and were found to reside in halos of $>10^{13} M_{\odot}$. These halo masses are also expected from the relation of stellar mass to halo mass (Behroozi et al. 2013, 2019; Durkalec et al. 2018). Astarte is about four times less massive than the average central galaxies at $z \sim 1$ of Hilton et al. (2013) and van der Burg et al. (2013), which indicates that these MS giant galaxies continue to grow either through in situ star formation or accretion of other galaxies throughout cosmic time until lower redshifts.

We compared Astarte with CO-detected samples of the same redshift range from Genzel et al. (2010, hereafter G10) and Tacconi et al. (2013, hereafter T13). These samples of SFGs have constrained $\mathrm{CO}$ detections and well-investigated physical parameters (in Sargent et al. 2014). 


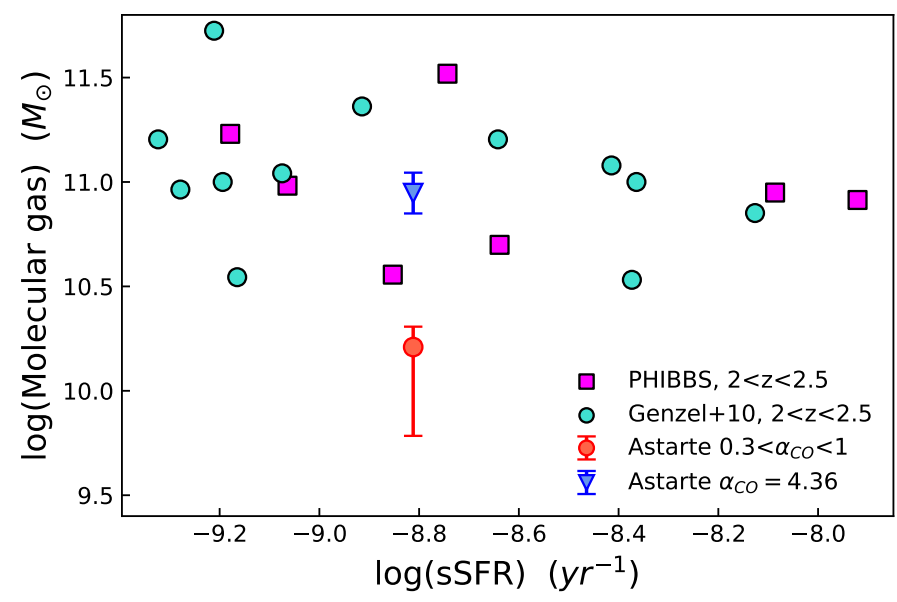

Fig. 6. Molecular gas masses derived with the $\mathrm{CO}$ conversion factor vs. the sSFR. The magenta squares are from T13. Turquoise circles are ULIRGs at $2<z<2.5$ from G10. The red circle shows the position of Astarte with $\alpha_{\mathrm{CO}}=0.8$, and the associated error bar shows the variation of the molecular gas mass using $0.3<\alpha_{\mathrm{CO}}<1$. The blue triangle shows the molecular gas of Astarte for a galactic $\mathrm{CO}$ conversion factor of 4.36 .

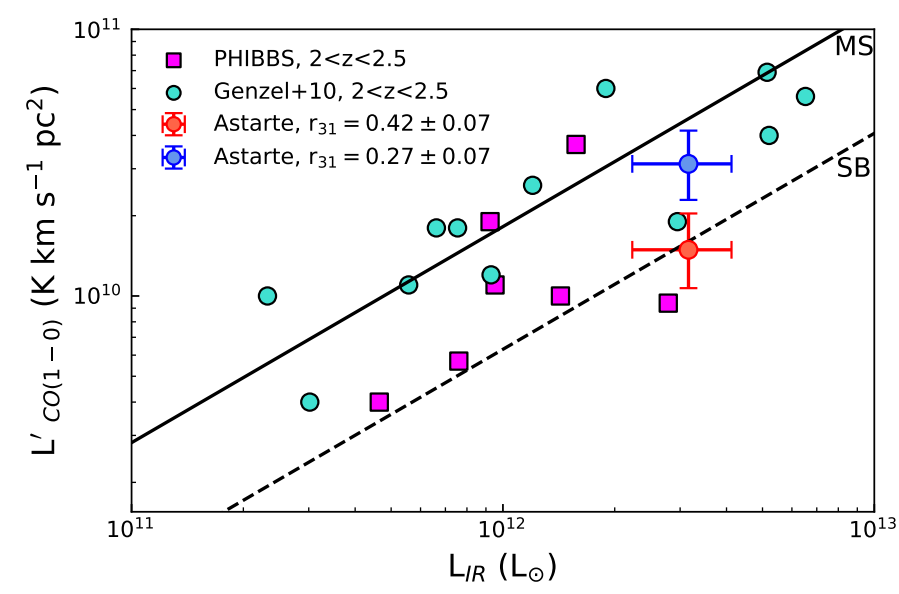

Fig. 7. Correlation between $\mathrm{CO}(1-0)$ luminosities and the total IR luminosity. The filled magenta squares are from T13 and filled turquoise circles are the sources from G10. The solid black line is the linear regression for MS galaxies (Sargent et al. 2014), and the dashed line is that for SBs (the regression lines are from the complete sample in Sargent et al. 2014).

We compared the molecular gas mass of Astarte derived from the $\mathrm{CO}$ emission line with that of $\mathrm{G} 10$ and T13 galaxies in Fig. 6. G10 used a galactic conversion factor for SFGs and $\alpha_{\mathrm{CO}}=1$ for ULIRGs, while T12 adopted a galactic conversion factor for all their sources. Our choice of $\alpha_{\mathrm{CO}}=0.8$ underestimates the molecular gas mass of Astarte compared to the galaxies of G10 and T13. However, $\alpha_{\mathrm{CO}}=4.36$ produces a higher molecular gas mass with respect to its sSFR.

In Fig. 7 we show the correlation between CO luminosities and the total IR luminosities. IR luminosities were derived from the SFRs of all the sources (G10, T13, and Astarte) using the Kennicutt relation (Kennicutt 1998). The initial choice of $r_{31}=$ $0.42 \pm 0.07$ (the average in Daddi et al. 2015) places Astarte on the SB line from Sargent et al. (2014), contradicting its SED result. We therefore investigated the lowest excitation ratio from Daddi et al. (2015) of $r_{31}=0.27 \pm 0.07$. This lower ratio moves Astarte closer to the MS within the error bars.
Using the total molecular gas mass of Astarte derived with the least excited $\mathrm{CO}(3-2)$ from Daddi et al. (2015) $\left(r_{31}=\right.$ $0.27 \pm 0.07)$, and assuming a galactic conversion factor, we estimate a gas fraction $f_{\text {gas }}=M_{\text {gas }} /\left(M_{\text {gas }}+M_{\star}\right)=(0.27 \pm 0.07)$. Although this falls within the lower limits of typical molecular gas fractions found in SFGs at $z \approx 2$ in Santini et al. (2014) and Béthermin et al. (2015), an $\alpha_{\mathrm{CO}}$ adapted for SB with a higher $r_{31}$ ratio reduces the gas fraction significantly.

The gas mass derived with a galactic conversion factor gives Astarte a rather short depletion time of $0.22 \pm 0.07 \mathrm{Gyr}$, making it very efficient at forming stars (for comparison, SBs have a depletion time of $\sim 100 \mathrm{Myr}$, see Fig. 10 in Béthermin et al. 2015). Recently, Elbaz et al. (2018) found that compact SFGs on the MS with a relatively short depletion time are common. These active ultramassive objects can be hidden at the higher end of the tail of the MS. The average depletion time for the Elbaz et al. (2018) galaxies is about $0.25 \mathrm{Gyr}$, and although we do not detect the continuum of Astarte with ALMA, its CO emission is compact, as it is the case for the continuum of ALMAdetected galaxies from Elbaz et al. (2018). This is also confirmed in Puglisi et al. (2019), where compact massive galaxies at the top of the MS exhibit high SFRs at their cores following their SB epoch.

\section{Conclusion}

We analyzed two galaxies, Astarte and Adonis, at the peak of the SFR density using multiwavelength dissection combining ALMA observations with UV-submillimeter SED modeling. We investigated the molecular gas content of Astarte through the ALMA detection of its $\mathrm{CO}(3-2)$ emission, relying on different excitation ratios of $L_{\mathrm{CO}(3-2)}^{\prime} / L_{\mathrm{CO}(1-0)}^{\prime}$ and different $\mathrm{CO}$ conversion factors. A galactic conversion factor when used along with the least excitation ratio from Daddi et al. (2015) confirmed the relative position of Astarte to the MS, as found from its SED modeling. Although the obtained gas fraction is at the lower limits of the fraction in MS galaxies (Santini et al. 2014; Béthermin et al. 2015), a possible explanation might be that the $\mathrm{CO}(3-2)$ instantaneous emission does not fully recover the molecular mass and the dynamics of Astarte because the excitation is weak (Daddi et al. 2015). Detections of other transition levels of $\mathrm{CO}$ would be helpful to better constrain the molecular mass of Astarte, and therefore its physical characteristics.

The physical dissociation of the CO line and the rest-frame stellar population in Astarte was also investigated, as was done in Buat et al. (2019), by deriving physical properties from the stellar emission (UV to near-IR) and the IR emission apart. As in Buat et al. (2019), the dust luminosity derived from the full SED agrees with the luminosity derived from the IR emission, while $L_{\text {dust }}$ derived from the stellar emission is slightly underestimated. Furthermore, the $\mathrm{C} 00$ attenuation law was preferred when the stellar continuum alone was fit. This is consistent with the results of Buat et al. (2019) for galaxies with the same radial extension of rest-frame stellar emission and ALMA-detected emission. The LF17 attenuation law, which was tuned for ULIRGs at $z \sim 2$, succeeds best in mimicking the dust attenuation of Astarte when the whole UV-submillimeter SED was fit; this results in a higher stellar mass, however.

The molecular mass of Astarte, obtained with a galactic conversion factor and the lowest excitation ratio from Daddi et al. (2015), contributes to $\sim 0.9$ of its total dynamical mass, which is a larger contribution than what was found in ULIRGs by Neri et al. (2003). SFRs and stellar masses derived from the SED fittings show that Adonis is a SB galaxy, while Astarte is on 
the MS of SFGs. However, the small gas fraction makes Astarte very efficient in forming stars; its depletion time is an order of magnitude lower than what is expected in typical MS galaxies (Béthermin et al. 2015). This SB-like star formation activity on the MS was found for massive compact SFGs in Puglisi et al. (2019) in their post-SB phase. Short depletion times of MS massive galaxies were also found by Elbaz et al. (2018), confirming that Astarte is caught in the middle of quenching following an earlier SB activity.

Central galaxies at $z \sim 1$ from Hilton et al. (2013) and van der Burg et al. (2013) are about four times more massive than Astarte. This indicates that even massive objects that are at the high end of the MS, when the Universe was undergoing its peak in the star formation rate density, continue their mass assembly down to lower redshifts.

Acknowledgements. M.H. and K.M. have been supported by the National Science Centre (UMO-2018/30/E/ST9/00082). We acknowledge and thank the referee for useful comments, which helped improving this work. This paper makes use of the following ALMA data: ADS/JAO.ALMA\#2013.1.00914.S. ALMA is a partnership of ESO (representing its member states), NSF (USA) and NINS (Japan), together with NRC (Canada), MOST and ASIAA (Taiwan), and KASI (Republic of Korea), in cooperation with the Republic of Chile. The Joint ALMA Observatory is operated by ESO, AUI/NRAO and NAOJ.

\section{References}

Alonso, M. S., Tissera, P. B., Coldwell, G., \& Lambas, D. G. 2004, MNRAS, 352,1081

Arnouts, S., \& Ilbert, O. 2011, Astrophysics Source Code Library [record ascl:1108.009]

Behroozi, P. S., Wechsler, R. H., \& Conroy, C. 2013, ApJ, 770, 57

Behroozi, P., Wechsler, R. H., Hearin, A. P., \& Conroy, C. 2019, MNRAS, 488, 3143

Béthermin, M., Kilbinger, M., Daddi, E., et al. 2014, A\&A, 567, A103

Béthermin, M., Daddi, E., Magdis, G., et al. 2015, A\&A, 573, A113

Béthermin, M., Wu, H.-Y., Lagache, G., et al. 2017, A\&A, 607, A89

Béthermin, M., Fudamoto, Y., Ginolfi, M., et al. 2020, A\&A, 643, A2

Blain, A. W., Smail, I., Ivison, R. J., Kneib, J. P., \& Frayer, D. T. 2002, Phys. Rep., 369, 111

Bolatto, A. D., Wolfire, M., \& Leroy, A. K. 2013, ARA\&A, 51, 207

Boquien, M., Burgarella, D., Roehlly, Y., et al. 2019, A\&A, 622, A103

Bothwell, M. S., Smail, I., Chapman, S. C., et al. 2013a, MNRAS, 429, 3047

Bothwell, M. S., Aguirre, J. E., Chapman, S. C., et al. 2013b, ApJ, 779, 67

Bourne, N., Dunlop, J. S., Merlin, E., et al. 2017, MNRAS, 467, 1360

Bruzual, G., \& Charlot, S. 2003, MNRAS, 344, 1000

Buat, V., Heinis, S., Boquien, M., et al. 2014, A\&A, 561, A39

Buat, V., Boquien, M., Małek, K., et al. 2018, A\&A, 619, A135

Buat, V., Ciesla, L., Boquien, M., Małek, K., \& Burgarella, D. 2019, A\&A, 632, A79

Burgarella, D., Buat, V., \& Iglesias-Páramo, J. 2005, MNRAS, 360, 1413

Calzetti, D., Armus, L., Bohlin, R. C., et al. 2000, ApJ, 533, 682

Carilli, C. L., \& Walter, F. 2013, ARA\&A, 51, 105

Casey, C. M., Scoville, N. Z., Sanders, D. B., et al. 2014, ApJ, 796, 95

Cassata, P., Morselli, L., Faisst, A., et al. 2020, A\&A, 643, A6

Cattaneo, A., Faber, S. M., Binney, J., et al. 2009, Nature, 460, 213

Chabrier, G. 2003, PASP, 115, 763

Chapman, S. C., Blain, A. W., Ivison, R. J., \& Smail, I. R. 2003, Nature, 422, 695

Chapman, S. C., Blain, A. W., Smail, I., \& Ivison, R. J. 2005, ApJ, 622, 772

Charlot, S., \& Fall, S. M. 2000, ApJ, 539, 718

Ciesla, L., Boquien, M., Boselli, A., et al. 2014, A\&A, 565, A128

Ciesla, L., Boselli, A., Elbaz, D., et al. 2016, A\&A, 585, A43

Ciesla, L., Elbaz, D., \& Fensch, J. 2017, A\&A, 608, A41

Ciesla, L., Elbaz, D., Schreiber, C., Daddi, E., \& Wang, T. 2018, A\&A, 615, A61

Coil, A. L., Newman, J. A., Croton, D., et al. 2008, ApJ, 672, 153
Combes, F. 2017, Front. Astron. Space Sci., 4, 10 Conroy, C. 2013, ARA\&A, 51, 393

da Cunha, E., Charlot, S., \& Elbaz, D. 2008, MNRAS, 388, 1595 Daddi, E., Elbaz, D., Walter, F., et al. 2010, ApJ, 714, L118

Daddi, E., Dannerbauer, H., Liu, D., et al. 2015, A\&A, 577, A46

Decarli, R., Walter, F., Gónzalez-López, J., et al. 2019, ApJ, 882, 138

Donevski, D., Lapi, A., Małek, K., et al. 2020, A\&A, 644, A144

Downes, D., \& Solomon, P. M. 1998, ApJ, 507, 615

Downes, D., \& Solomon, P. M. 2003, ApJ, 582, 37

Draine, B. T., \& Li, A. 2007, ApJ, 657, 810

Draine, B. T., Aniano, G., Krause, O., et al. 2014, ApJ, 780, 172

Dubois, Y., Gavazzi, R., Peirani, S., \& Silk, J. 2013, MNRAS, 433, 3297

Dunlop, J. S., McLure, R. J., Biggs, A. D., et al. 2017, MNRAS, 466, 861

Durkalec, A., Le Fèvre, O., Pollo, A., et al. 2018, A\&A, 612, A42

Elbaz, D., Leiton, R., Nagar, N., et al. 2018, A\&A, 616, A110

Faisst, A. L., Schaerer, D., Lemaux, B. C., et al. 2020, ApJS, 247, 61

Genzel, R., Tacconi, L. J., Gracia-Carpio, J., et al. 2010, MNRAS, 407, 2091

González-López, J., Novak, M., Decarli, R., et al. 2020, ApJ, 897, 91

Greve, T. R., Bertoldi, F., Smail, I., et al. 2005, MNRAS, 359, 1165

Gruppioni, C., Béthermin, M., Loiacono, F., et al. 2020, A\&A, 643, A8

Hainline, L. J., Blain, A. W., Smail, I., et al. 2011, ApJ, 740, 96

Hilton, M., Hasselfield, M., Sifón, C., et al. 2013, MNRAS, 435, 3469

Hopkins, A. M., \& Beacom, J. F. 2006, ApJ, 651, 142

Jin, S., Daddi, E., Liu, D., et al. 2018, ApJ, 864, 56

Kennicutt, R. C., Jr 1998, ARA\&A, 36, 189

Komatsu, E., Smith, K. M., Dunkley, J., et al. 2011, ApJS, 192, 18

Laigle, C., McCracken, H. J., Ilbert, O., et al. 2016, ApJS, 224, 24

Lambas, D. G., Tissera, P. B., Alonso, M. S., \& Coldwell, G. 2003, MNRAS, 346, 1189

Law, D. R., Steidel, C. C., Erb, D. K., et al. 2009, ApJ, 697, 2057

Lilly, S. J., Le Brun, V., Maier, C., et al. 2009, ApJS, 184, 218

Lo Faro, B., Buat, V., Roehlly, Y., et al. 2017, MNRAS, 472, 1372

Lutz, D., Poglitsch, A., Altieri, B., et al. 2011, A\&A, 532, A90

Madau, P., \& Dickinson, M. 2014, ARA\&A, 52, 415

Małek, K., Buat, V., Roehlly, Y., et al. 2018, A\&A, 620, A50

McMullin, J. P., Waters, B., Schiebel, D., Young, W., \& Golap, K. 2007, in Astronomical Data Analysis Software and Systems XVI, eds. R. A. Shaw, F. Hill, \& D. J. Bell, ASP Conf. Ser., 376, 127

Mendez, A. J., Coil, A. L., Lotz, J., et al. 2011, ApJ, 736, 110

Merlin, E., Fontana, A., Castellano, M., et al. 2018, MNRAS, 473, 2098

Michałowski, M. J., Dunlop, J. S., Cirasuolo, M., et al. 2012, A\&A, 541, A85

Murphy, E. J., Chary, R. R., Dickinson, M., et al. 2011, ApJ, 732, 126

Neri, R., Genzel, R., Ivison, R. J., et al. 2003, ApJ, 597, L113

Noeske, K. G., Weiner, B. J., Faber, S. M., et al. 2007, ApJ, 660, L43

Noll, S., Burgarella, D., Giovannoli, E., et al. 2009, A\&A, 507, 1793

Oliver, S. J., Bock, J., Altieri, B., et al. 2012, MNRAS, 424, 1614

Puglisi, A., Daddi, E., Liu, D., et al. 2019, ApJ, 877, L23

Riechers, D. A., Boogaard, L. A., Decarli, R., et al. 2020, ApJ, 896, L21

Rodighiero, G., Daddi, E., Baronchelli, I., et al. 2011, ApJ, 739, L40

Salim, S., \& Narayanan, D. 2020, ARA\&A, 58, 529

Santini, P., Maiolino, R., Magnelli, B., et al. 2014, A\&A, 562, A30

Sargent, M. T., Daddi, E., Béthermin, M., et al. 2014, ApJ, 793, 19

Schmidt, M. 1959, ApJ, 129, 243

Schreiber, C., Pannella, M., Elbaz, D., et al. 2015, A\&A, 575, A74

Schreiber, C., Labbé, I., Glazebrook, K., et al. 2018a, A\&A, 611, A22

Schreiber, C., Glazebrook, K., Nanayakkara, T., et al. 2018b, A\&A, 618, A85

Smail, I., Ivison, R. J., \& Blain, A. W. 1997, ApJ, 490, L5

Smolčić, V., Novak, M., Bondi, M., et al. 2017, A\&A, 602, A1

Solomon, P. M., Downes, D., Radford, S. J. E., \& Barrett, J. W. 1997, ApJ, 478, 144

Tacconi, L. J., Neri, R., Chapman, S. C., et al. 2006, ApJ, 640, 228

Tacconi, L. J., Neri, R., Genzel, R., et al. 2013, ApJ, 768, 74

Takeuchi, T. T., Buat, V., \& Burgarella, D. 2005, A\&A, 440, L17

van der Burg, R. F. J., Muzzin, A., Hoekstra, H., et al. 2013, A\&A, 557, A15

Wang, T., Schreiber, C., Elbaz, D., et al. 2019, Nature, 572, 211

Weiß, A., De Breuck, C., Marrone, D. P., et al. 2013a, ApJ, 767, 88

Weiß, A., De Breuck, C., Vieira, J. D., et al. 2013b, in New Trends in Radio Astronomy in the ALMA Era: The 30th Anniversary of Nobeyama Radio Observatory, eds. R. Kawabe, N. Kuno, \& S. Yamamoto, ASP Conf. Ser., 476,33

Whitaker, K. E., Pope, A., Cybulski, R., et al. 2017, ApJ, 850, 208

Wild, V., Charlot, S., Brinchmann, J., et al. 2011, MNRAS, 417, 1760 